\title{
The effect of tibolone in postmenopausal women receiving tamoxifen after surgery for breast cancer: a randomised, double-blind, placebo-controlled trial
}

\author{
R. Kroiss, ${ }^{a}$ I.S. Fentiman, ${ }^{b}$ F.A. Helmond, ${ }^{c}$ J. Rymer, ${ }^{b}$ J.M. Foidart, ${ }^{\mathrm{d}}$ N. Bundred, ${ }^{\mathrm{e}}$ M. Mol-Arts, ${ }^{\mathrm{c}}$ E. Kubista ${ }^{\mathrm{a}}$ \\ ${ }^{a}$ Medical University of Vienna and Ludwig Boltzmann Institute for Clinical Experimental Oncology, Austria \\ ${ }^{b}$ Guys Hospital, London, UK \\ ${ }^{c}$ NV Organon, Oss, The Netherlands \\ ${ }^{d}$ Center Hospitalier Regional de la Citadelle, Liège, Belgium \\ ${ }^{e}$ South Manchester University Hospital, UK
}

Objective To assess the effects of tibolone on climacteric symptoms, endometrium and serum lipid/ lipoproteins in postmenopausal women receiving tamoxifen after surgery for breast cancer.

Design Double-blind, randomised, placebo-controlled, multicentre pilot study.

Setting Hospital outpatient clinic.

Sample Seventy postmenopausal women receiving tamoxifen following surgery for early breast cancer.

Methods Women received $20 \mathrm{mg}$ /day oral tamoxifen plus either $2.5 \mathrm{mg} /$ day oral tibolone or placebo for 12 months.

Main outcome measures Frequency and severity of hot flushes (diary cards); intensity of hot flushes and sweats (Landgren scale); interference of hot flushes and sweats with normal life; frequency and intensity of other climacteric symptoms; endometrial thickness and histology; vaginal bleeding; breast cancer recurrence and serum lipid/lipoproteins.

Results Daily card data showed no change in the daily number of hot flushes with either tibolone or placebo $(P=0.219)$ after three months. There was a significant reduction in the severity of flushes with tibolone compared with placebo $(-0.4$ vs $0.2, P=0.031)$. The Landgren scale showed a mean change in the number of hot flushes of -0.6 with tibolone and +1.1 with placebo after 12 months $(P=0.022)$. Endometrial biopsies were normal and vaginal bleeding was similar in both groups. A significant decrease in triglycerides (-23\% vs $1.4 \%)$ and HDL (-12\% vs 19\%) was seen with tibolone compared with placebo after 12 months.

Conclusions Tibolone prevented an increase in hot flushes in postmenopausal women given tamoxifen following surgery for breast cancer without untoward effects on the endometrium. Beneficial effects on serum lipid profile were noted.

\section{INTRODUCTION}

Breast cancer is the most common cancer among women in Western countries. Approximately two-thirds of cases occur in postmenopausal women, and hence, many will suffer from climacteric symptoms. Moreover, the majority will be given adjuvant tamoxifen that may exacerbate these symptoms and lead to discontinuation of adjuvant therapy. ${ }^{1}$ Combined oestrogen and progestogen therapy has conventionally been contraindicated in these women due to fears that oestrogen might stimulate the growth of residual micro-metastases leading to recurrence of breast cancer. Although there is no clear evidence of such an effect, the issue of breast cancer and oestrogen and progestogen therapy remains highly controversial. Some studies have suggested a reduced incidence of recurrence and mortality in breast cancer survivors given oestrogen and progestogen therapy, ${ }^{3}$ but the prematurely stopped HABITS and Stockholm trials did not resolve the debate.

Tibolone is a drug that has beneficial effects on climacteric symptoms, bone, the vagina, and mood and sexual wellbeing ${ }^{5}$ due to the effects of its two oestrogenic metabolites and a third metabolite with androgenic properties. ${ }^{6}$ This third metabolite also exerts local progestogenic effects in the endometrium and prevents endometrial stimulation. ${ }^{7}$ Tamoxifen acts as an oestrogen agonist on endometrial cells, ${ }^{8}$ and causes endometrial stimulation which may lead to endometrial hyperplasia or cancer. ${ }^{9}$

Tibolone and its metabolites have a tissue-selective effect in the breast, inhibiting sulphatase activity to prevent formation of active oestrogens and stimulating sulpho-transferase to encourage formation of inactive sulphated oestrogens. Tibolone stimulates apoptosis and reduces proliferation rate in normal human breast tissues in vitro and in vivo. ${ }^{11}$ Animal studies have shown that it does not stimulate mammary tissue ${ }^{12}$ and displays an antitumour effect on the breast similar to that of tamoxifen. ${ }^{13}$ 
These findings suggest that tibolone might be beneficial in reducing climacteric symptoms while preventing endometrial stimulation associated with tamoxifen. A double-blind, placebo-controlled study was therefore conducted to assess whether tibolone could prevent, relieve or delay the occurrence of climacteric symptoms in postmenopausal women treated with tamoxifen following breast cancer surgery.

\section{METHODS}

Postmenopausal women (hospital outpatients; $\leq 75$ years old; body mass index $18-30 \mathrm{~kg} / \mathrm{m}$ ) with newly diagnosed and histologically confirmed invasive or non-invasive early stage breast cancer ( $\leq$ stage IIb), for which they were to receive surgical treatment (conservation therapy or modified radical mastectomy) followed by tamoxifen, were enrolled in the study. The women were required to have had their last natural menstrual period $\geq 1$ year before diagnosis of breast cancer and to have a serum oestradiol concentration of $\leq 30 \mathrm{pg} / \mathrm{mL}$. Exclusion criteria included other malignancies, prior hysterectomy and/or bilateral oophorectomy, endometrial hyperplasia/adenocarcinoma, cervical smear result showing moderate dysplasia or worse, cardiovascular, cerebrovascular or thromboembolic disorders, uterine bleeding of unknown cause, severe liver disorders, drug or alcohol abuse in the previous 12 months, requirement for cancer therapy (apart from tamoxifen or radiotherapy) or medication that might affect the metabolism of tibolone, and the use of steroids or tamoxifen in the six weeks prior to the study or hormonal implants at any time. All women provided written informed consent. The protocol was approved by the Independent Ethics Committee of each study centre.

Women were randomised to receive $20 \mathrm{mg}$ /day oral tamoxifen (Nolvadex-D; Astra-Zeneca) plus either $2.5 \mathrm{mg} /$ day oral tibolone (Livial; NV Organon) or matching placebo. Medication was started within three days of the baseline assessment (which was performed within 12 weeks of breast surgery) and was continued for 12 months. Compliance was checked by means of a daily diary and inquiry by the investigator. The automated random assignment of subject numbers to treatment groups was made by the Clinical Supply Management at Organon, using the Almedica Drug Labeling System (ADLS); the medication was then coded and labelled at Organon and dispensed by the investigator. Randomisation was done in blocks of four. Tibolone and placebo tablets were identical in shape, appearance and packaging. Assessments were performed at screening, baseline, three-monthly intervals during treatment and on discontinuation from the study.

The women were screened with a medical, gynaecological and drug history, physical examination, gynaecological examination and cervical cytology, endometrial biopsy and routine laboratory analyses. Information on mammography results, site of breast malignancy, breast status after surgery, nodal status of the tumour, ratio of nodes containing metastases and oestrogen and progestogen receptor status of the malignancy were documented at baseline.

The primary efficacy parameters were the frequency and severity of hot flushes at three months. This was documented by the patient using a seven-day diary card between the screening and baseline assessments and during the first three months of treatment. The severity of hot flushes was rated as mild (sensation of heat without perspiration), moderate (sensation of heat with sweating but able to continue activity) or severe (sensation of heat with sweating and unable to continue activity). These parameters are in accordance with the Food and Drug Administration guidance and have been used previously. ${ }^{14}$ In order to determine the average daily severity of hot flushes, a weighted average score was calculated for each patient: [(Number of mild hot flushes x 1) $+($ Number of moderate hot flushes x 2$)+($ Number of severe hot flushes x 3$)] /$ Total number of hot flushes.

Climacteric symptoms (hot flushes, sweats, palpitations, joint pain, vaginal dryness and incontinence) were also recorded on a checklist, adapted from the Landgren scale, ' at baseline and each visit. This checklist was completed by the investigator and reflected the patient's symptom recall over the previous week. Intensity was classified as: none (no symptoms), mild (not interfering with daily activities or sleep), moderate (interfering with daily activities or sleep, but these activities could be continued), severe (interfering with daily activities or sleep and stopping them for up to 5 minutes) or very severe (interfering with daily activities or sleep and stopping them for more than 5 minutes).

Secondary efficacy parameters included interference of hot flushes and sweats with everyday life (none, mild, moderate, severe or very severe) and the intensity of palpitations, joint pain, vaginal dryness (none, mild, moderate, severe or very severe), all of which were assessed using a validated questionnaire. ${ }^{15,16}$

Vital signs and routine laboratory parameters were measured before baseline and at each visit, together with a clinical examination of the breasts. A gynaecological examination and an endometrial biopsy were performed 
after 6 and 12 months and a physical examination and mammography after 12 months. A monthly diary card was used to document the incidence of bleeding/spotting throughout the study. Adverse events were recorded throughout the study. A serious adverse event was defined as any event that was fatal or life-threatening, permanently disabling or required inpatient hospitalisation.

The primary safety parameters were the incidence of vaginal bleeding/spotting and endometrial histology. Endometrial biopsies were classified as normal (no tissue obtained, tissue insufficient, atrophic/inactive, secretory, menstrual type or proliferative endometrium); abnormal (hyperplastic endometrium, carcinoma or other); or polyps. Secondary safety parameters comprised the serum lipid/ lipoprotein profile and the recurrence of breast cancer.

The rationale for the sample size was based on data showing that after three months of tamoxifen treatment, up to $65 \%$ of the subjects experienced frequent and severe hot flushes. ${ }^{17}$ Assumptions were made concerning the expected proportions under combined tamoxifen and tibolone and the required number of subjects was calculated using a $\chi^{2}$ test. The working hypothesis was that, under combined tamoxifen and tibolone, this proportion would be a maximum of $30 \%$, thus leading to an effective sample size of 32 per group. A difference between groups of about 0.7 times the standard deviation could be detected in the number of hot flushes $(\alpha=0.05$ two-tailed, $1-\beta=$ 0.80 power).

Data were analysed on an intention-to-treat basis. Group comparisons were carried out using $\chi^{2}$ or Fisher's exact tests for dichotomous variables and non-parametric analysis for continuous variables. Data are presented as mean (standard deviation).

Fig. 1: Progress through the study.

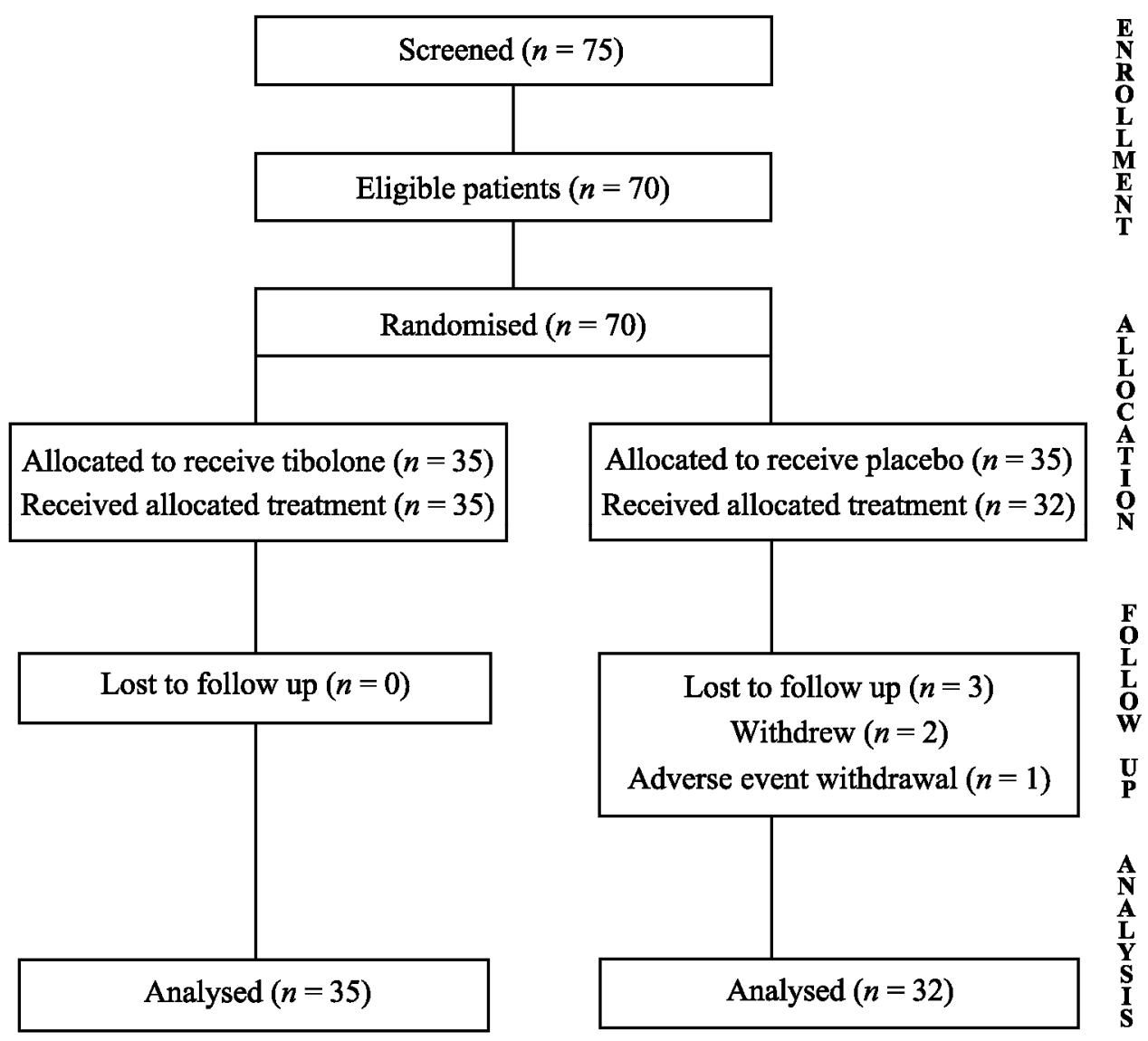

\section{RESULTS}

A total of 75 women were screened between July 1996 and July 2000, of whom 70 were randomised to treatment, 35 in each group. All tibolone-treated patients completed the study, while three women in the placebo 
group withdrew prematurely [one due to an adverse event (aggravation of Parkinson's disease) and two who were unwilling/unable to co-operate further] (Fig. 1). The baseline characteristics were similar in both groups (Table 1).

The total exposure in the tibolone group was 35.1 woman years and in the placebo group 32.9 woman years. Dosing compliance was very high for both tibolone (99.5\%) and placebo (97.8\%). Almost 100\% compliance was also seen with tamoxifen.

Baseline data for the daily number of hot flushes were incomplete or missing for 16 women (tibolone, $n=7$; placebo, $n=9$ ). As hot flushes were not an entry requirement for the study, baseline data on severity were not available for a considerable number of women (tibolone, $n=19$; placebo, $n=23$ ). More women in the tibolone (78.6\%) than in the placebo group (50\%) experienced hot flushes at baseline. The changes in number and severity of hot flushes recorded on diary cards are shown in Table 2.

Using the Landgren scale, more women in the tibolone group than in the placebo group experienced hot flushes $(72.7 \%$ vs $39.4 \%)$ and sweats $(54.5 \%$ vs $36.4 \%)$ at baseline. Thirty-three women in the tibolone group and 31 in the placebo group had baseline and final visit data available. There was a decrease of approximately $30 \%$ in the occurrence of hot flushes and sweats with tibolone and an increase of approximately 30\% with placebo after 12 months of treatment. The mean change from baseline in the number of hot flushes was -0.6 with tibolone and + 1.1 with placebo $(P=0.022)$; the corresponding changes in the number of sweats were -0.6 and 1.2 , respectively $(P=0.22)$. The mean change in intensity with tibolone and placebo was -0.2 and +0.2 , respectively $(P=0.09)$, for hot flushes and -0.2 and +0.3 , respectively $(P=0.15)$, for sweats.

The percentage of women in whom hot flushes and sweats did not interfere with everyday life increased from $52 \%$ to $74 \%$ at 12 months in the tibolone group $(P=0.071)$ and from $64 \%$ to $70 \%$ in the placebo group $(P=0.48)$. Only minor changes were seen in the other climacteric symptoms, with no significant differences between the groups (data not shown).

Biopsies were obtained from 35 women in the tibolone group and 32 in the placebo group at baseline and 33 in the tibolone group and 30 women in the placebo groups at 12 months. All biopsies were defined as normal. The results were mainly 'atrophic/inactive' in the tibolone group (one woman with secretory endometrium) and 'tissue insufficient' in the placebo group (one women with proliferative endometrium). An increase in endometrial thickness occurred in both groups during the first three months. Although the increase was more marked in the tibolone group at the start of the study, the endometrial thickness was similar in both groups from month nine onwards (data not shown). Three women (one in the tibolone group and two in the placebo group) underwent hysteroscopy and curettage but no evidence of malignancy was detected.

Vaginal bleeding or spotting occurred in 10 women (28.6\%) receiving tibolone and 7 women (20.6\%) receiving placebo $(P=0.44)$. The mean duration of bleeding was $3.9(3.8)$ days with tibolone and $2.7(2.6)$ with placebo (not significant). There were no differences in the number of episodes or timing of bleeding between the groups.

Table 1: Baseline characteristics of study patients. Values are presented as mean [SD] or n (\%).

\begin{tabular}{lcc} 
& $\begin{array}{c}\text { Tibolone } \\
(n=35)\end{array}$ & $\begin{array}{c}\text { Placebo } \\
(n=35)\end{array}$ \\
\hline Age (years) & $58[6.1]$ & $59[5.5]$ \\
Body weight $(\mathrm{kg})$ & $65[9]^{\mathrm{a}}$ & $68[11]$ \\
Height $(\mathrm{cm})$ & $162[6.8]$ & $163[6.5]$ \\
Body mass index $\left(\mathrm{kg} / \mathrm{m}^{2}\right)$ & $24.8[3.2]^{\mathrm{a}}$ & $25.4[3.7]$ \\
Time since menopause $(\mathrm{months})$ & $105[75]^{\mathrm{a}}$ & $109[61]^{\mathrm{a}}$ \\
Median tumour size $(\mathrm{mm})$ & $13.5^{\mathrm{a}}$ & 14.0 \\
Patients with & & \\
Positive axillary nodes & $6(17)$ & $7(20)$ \\
Positive oestrogen receptor & $28(85)^{\mathrm{b}}$ & $34(97)$ \\
Positive progestogen receptor & $22(68)^{\mathrm{c}}$ & $23(68)^{\mathrm{a}}$ \\
\hline
\end{tabular}

$$
\begin{aligned}
& { }^{a} n=34 . \\
& { }^{\mathrm{b}} n=33 .
\end{aligned}
$$

${ }^{\mathrm{c}} n=32$. 
Table 2: Daily number and severity of hot flushes at baseline and after three months of treatment, and change from baseline (diary cards). Values are presented as mean [SD].

\begin{tabular}{lccc} 
& Tibolone & Placebo & $P$ \\
\hline No. of hot flushes & & & \\
Baseline & $2.0[2.1]^{\mathrm{a}}$ & $2.2[3.3]^{\mathrm{b}}$ & \\
Last week & $2.1[2.1]^{\mathrm{c}}$ & $2.9[3.2]^{\mathrm{d}}$ & 0.66 \\
Change from baseline & $-0.1[1.9]^{\mathrm{b}}$ & $0.9[3.4]^{\mathrm{b}}$ & 0.22 \\
Severity of hot flushes* & & & \\
Baseline & $1.6[0.6]^{\mathrm{e}}$ & $1.6[0.5]^{\mathrm{f}}$ & \\
Last week & $1.4[0.3]^{\mathrm{e}}$ & $1.7[0.6]^{\mathrm{g}}$ & 0.11 \\
Change from baseline & $-0.4[0.7]^{\mathrm{h}}$ & $0.2[0.4]^{\mathrm{h}}$ & 0.03 \\
\hline${ }^{\mathrm{a}} n=28$. & & \\
${ }^{\mathrm{b}} n=26$. & & \\
${ }^{\mathrm{c}} n=30$. & & \\
${ }^{\mathrm{d}} n=32$. & & \\
${ }^{\mathrm{e}} n=16$. & & \\
${ }^{\mathrm{f}} n=12$. & & \\
${ }_{\mathrm{g}} \mathrm{g} n=17$. & & \\
${ }^{\mathrm{h}} n=8$. & & \\
${ }^{*}$ Weighted average score. & & & \\
& & &
\end{tabular}

Table 3: Baseline values for lipids and lipoproteins parameters and percentage change from baseline at 12 months. Values are presented as mean [SD].

\begin{tabular}{lccc} 
& Tibolone & Placebo & $P$ \\
\hline Triglycerides (mmol/L) & & & \\
Baseline & $1.7[1.1]^{\mathrm{a}}$ & $1.7[0.8]^{\mathrm{b}}$ & \\
\% Change at 12 months & $-23[27]^{\mathrm{c}}$ & $1.4[36]^{\mathrm{d}}$ & 0.005 \\
Lipoprotein(a) (U/L) & & & \\
Baseline & $292[358]^{\mathrm{c}}$ & $254[342]^{\mathrm{c}}$ & \\
\% Change at 12 months & $-14[83]^{\mathrm{c}}$ & $7[120]^{\mathrm{e}}$ & 0.46 \\
LDL (mmol/L) & & & \\
Baseline & $3.8[0.9]^{\mathrm{a}}$ & $4.1[1.0]^{\mathrm{b}}$ & \\
\% Change at 12 months & $4.8[31]^{\mathrm{c}}$ & $-15[14]^{\mathrm{d}}$ & 0.0029 \\
HDL (mmol/L) & & & \\
Baseline & $1.4[0.4]^{\mathrm{a}}$ & $1.3[0.3]^{\mathrm{b}}$ & \\
\% Change at 12 months & $-12[22]^{\mathrm{c}}$ & $19[26]^{\mathrm{d}}$ & $<0.001$
\end{tabular}

\section{Cholesterol total (mmol/L)}

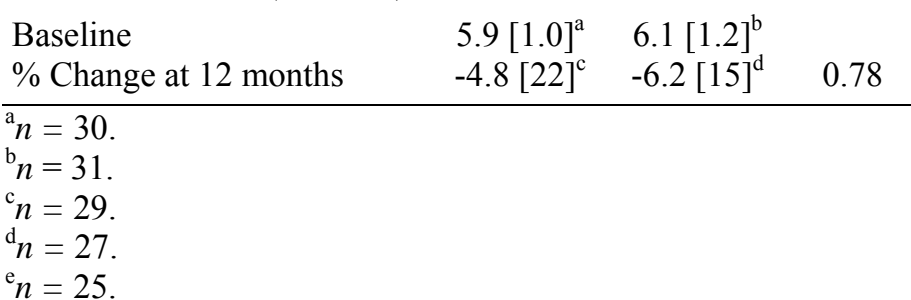

Table 4: Adverse events. Values are presented as $n(\%)$.

\begin{tabular}{lcc}
\hline & Tibolone & Placebo \\
\hline Erythema & $10(29)$ & $10(29)$ \\
Skin disorder & $1(2.9)$ & $4(11)$ \\
Joint pain & $6(17)$ & $1(2.9)$ \\
Weight increase & $4(11)$ & $7(20)$ \\
Vaginitis & $6(17)$ & $2(5.7)$ \\
Back pain & $2(5.7)$ & $5(14)$ \\
Influenza-like symptoms & $1(2.9)$ & $4(11)$ \\
Oedema & $4(11)$ & $3(8.6)$ \\
\hline
\end{tabular}

In the tibolone-treated patients, a significant decrease in triglycerides (-23\% vs $1.4 \%)$ and HDL (-12\% vs $19 \%)$ 
could be observed after 12 months of treatment (see Table 3).

None of the women in either group experienced a recurrence of breast cancer. Mammography results and breast examinations did not reveal any clinically significant findings. Adverse events were reported by 25 women $(71 \%)$ in the tibolone group and $29(83 \%)$ in the placebo group (Table 4$)$.

\section{DISCUSSION}

In this study, we have demonstrated that tibolone prevented the increase in the occurrence, severity and intensity of hot flushes and sweats associated with tamoxifen treatment following surgical treatment for breast cancer. There was also a trend for tibolone to reduce the impact of hot flushes and sweats on normal daily life. Tibolone has been used effectively for the management of climacteric symptoms in a previous small study involving 15 postmenopausal women with breast cancer who had undergone surgery and radiotherapy. As in the current study, none of the patients suffered a recurrence of breast cancer and eight were still being treated with tibolone one year after the end of the study.

Clinical evidence indicates that tibolone is associated with a lower incidence of breast tenderness than conventional oestrogen replacement therapy ${ }^{19-22}$ and that it does not appear to change breast density. ${ }^{11,22-26}$ For example, in a six-month, double-blind, placebo-controlled study in 166 healthy postmenopausal women, $46 \%$ of those taking continuous combined oestrogen replacement therapy had an increase in breast density (Wolfe classification) compared with $2 \%$ taking tibolone and $0 \%$ receiving placebo. ${ }^{22}$ Increased density can impair interpretation of mammograms and thus reduce the sensitivity of screening programmes to detect breast cancer. ${ }^{27}$ These observations support preclinical research indicating that tibolone does not exert an oestrogenic effect on the breast. ${ }^{10,12,13}$ Nevertheless, the clinical significance of these findings is currently unclear and the relationship between tibolone and breast cancer will require further evaluation.

The recent Million Women Study, which recruited over 1 million women aged 50-64 years, reported that all types of hormone therapy were associated with an increased risk of breast cancer, this was substantially greater for oestrogen and progestogen therapy than for tibolone and oestrogen alone. ${ }^{28}$ The proportion of tibolone users was small $(6 \%)$ and it is unclear whether these women carried risk factors other than those controlled for in the analyses. ${ }^{29-31}$ In contrast to the Million Women Study, a large case-control study in the UK General Physicians Database, with a similar number of breast cancer cases, did not reveal an increased risk for breast cancer in women treated with tibolone. ${ }^{32}$

The reported increased risk of breast cancer with oestrogen containing hormone therapy may be partially due to accelerated tumour growth, a factor which is clearly not applicable to women who have had surgery. However, the growth of micro-metastases may be enhanced leading to an increased recurrence rate. Two recent (prematurely stopped) randomised trials provided conflicting information. The HABITS Trial reported an increased risk for new breast cancer events which was not confirmed in the Stockholm Trial. ${ }^{4}$ The effect of tibolone on the breast recurrence rate in women with a history of breast cancer is currently being assessed in a large double-blind, randomised, placebo-controlled, five-year clinical trial (LIBERATE).

Endometrial safety is an important issue in women receiving tamoxifen treatment. ${ }^{9}$ As expected, ${ }^{33}$ there was an initial increase in endometrial thickness in both groups in our study. Nevertheless, the endometrial biopsies were all normal. The endometrial safety of tibolone has been previously established, ${ }^{5}$ and the current findings indicate that this profile is maintained in women receiving tamoxifen treatment. The effects of tibolone on serum lipid and lipoprotein levels were in line with those from previous reports and therefore appear to be unaffected by concomitant tamoxifen treatment.

At present, there are no approved effective compounds to treat postmenopausal women with a history of breast cancer who are suffering from climacteric symptoms. The findings from this pilot study are promising, but only a large, long term clinical trial can provide confirmatory data regarding the effects of tibolone on recurrence in breast cancer patients.

\section{References}

1. Fentiman IS, Howell A, Hamed H, et al. A controlled trial of adjuvant tamoxifen, with or without prednisolone, in postmenopausal women with operable breast cancer. Br J Cancer 1994;70:729-731. 
3. O'Meara ES, Rossing MA, Dalling JR, Elmore JG, Barlow WE, Weiss NS. Hormone replacement therapy after a diagnosis of breast cancer in relation to recurrence and mortality. J Natl Cancer Inst 2001;93:754-762.

4. Holmberg L, Anderson H. HABITS (hormonal replacement therapy after breast cancer-is it safe?) a randomised comparison: trial stopped. Lancet 2004;363:453-455.

5. Moore RA. Livial: a review of clinical studies. Br J Obstet Gynaecol 1999;106(Suppl 19):1-21.

6. Kloosterboer HJ. Tibolone: a steroid with a tissue-specific mode of action. J Steroid Biochem Mol Biol 2001;76:231-238.

7. Markiewicz L, Gurpride E. In vitro evaluation of estrogenic, estrogen antagonistic and progestogenic effects of a steroidal drug (Org OD14) and its metabolites on human endometrium. J Steroid Biochem 1990; 35:535-541.

8. Gusberg SB. Tamoxifen for breast cancer: associated endometrial cancer. Cancer 1990;65:1463-1464.

9. van Leeuwen FF, Benraadt J, Coeberger WW, et al. Risk of endometrial cancer after tamoxifen treatment of breast cancer. Lancet $1994 ; 343: 448-452$.

10. Compel A, Chaouat M, Jacob D, Perrot J-Y, Kloosterboer HJ, Rostene W. In vitro studies of tibolone in breast cells. Fertil Steril 2002;78:351-359.

11. Valdivia I, Campodónico I, Tapia A, Capetillo M, Espinoza A, Lavín P. Effects of tibolone and continuous combined hormone therapy on mammographic breast density and breast histochemical markers in postmenopausal women. Fertil Steril 2004;81:617-623.

12. Cline JM, Anthony MS, Register TC, Borgerink H, Clarkson TB. Tibolone does not stimulate the endometrium or breast in cynomolgus monkeys. FASEB J 2001;15:A594.

13. Kloosterboer HJ, Schoonen WG, Deckers GH, Klijn JG. Effects of progestagens and Org OD14 in in vitro and in vivo tumor models. $J$ Steroid Biochem Mol Biol 1994;49:311-318.

14. Utian WH, Shoupe D, Bachmann G, Pinkerton JV, Pickar JH. Relief of vasomotor symptoms and vaginal atrophy with lower doses of conjugated equine estrogens and medroxyprogesterone acetate. Fertil Steril 2001;75:1065-1079.

15. Collins A, Landgren BM. Psychosocial factors associated with the use of hormonal replacement therapy in a longitudinal follow-up of Swedish women. Maturitas 1997;28:1-9.

16. Landgren MB, Bennink HJ, Helmond FA, Engelen S. Dose-response analysis of effects of tibolone on climacteric symptoms. Br J Obstet Gynaecol 2002;109:1109-1114.

17. Love RR, Cameron L, Connell BL, Leventhal H. Symptoms associated with tamoxifen treatment in postmenopausal women. Arch Intern Med 1991;151:1842-1847.

18. Ginsburg J, Prelevic G. Tibolone in postmenopausal women with a history of breast carcinoma. J Br Menopause Soc 1995;1:24-25.

19. Dören M, Rübig A, Coelingh Bennink HJT, Holzgreve W. Impact on uterine bleeding and endometrial thickness: tibolone compared with continuous combined estradiol and norethisterone acetate replacement therapy. Menopause 1999;6:299-306.

20. Huber J, Palacios S, Berglund L, et al. The effect of tibolone compared with conjugated equine oestrogens continuously combined with medroxyprogesterone acetate on bleeding rates, quality of life and tolerability in postmenopausal women. Br J Obstet Gynaecol 2002; 109:886-893

21. Hammar M, Christau S, Nathorst-Böös J, Rud T, Carre K. A double-blind randomised trial comparing the effects of tibolone and continuous combined hormone replacement therapy in postmenopausal women with menopausal symptoms. Br J Obstet Gynaecol 1998;105:904-911.

22. Lundström E, Christow A, Svane G, et al. Effects of tibolone and a continuous combined HRT regimen on mammographic breast density. Am J Obstet Gynecol 2002;186:717-722.

23. Valdivia I, Ortega D. Mammographie density in postmenopausal women treated with tibolone, estriol or conventional hormone replacement therapy. Clin Drug Invest 2000;20:101-107.

24. Erel CT, Esen G, Seyisoglu H, et al. Mammographie density increase in women receiving different hormone replacement regimens. Maturitas 2001;40:151-157.

25. Colacurci N, Fornaro F, de Franciscis P, Palermo M, del Vecchio E. Effects of different types of hormone replacement therapy on mammographie density. Maturitas 2001;40:159-164.

26. Egarter C, Eppel W, Vogel S, Wolf G. A pilot study of hormone replacement therapy with tibolone in women with mastopathic breasts. Maturitas 2001;40:165-171. 
Published in : BJOG : An International Journal of Obstetrics \& Gynaecology (2005), vol. 112, iss. 2, pp 228-233 Status : Postprint (Author's version)

27. Kavanagh AM, Mitichell H, Giles GG. Hormone replacement therapy and accuracy of mammographie screening. Lancet 2000; $355: 270-274$

28. Million Women Study Collaborators. Breast cancer and hormone replacement therapy in the Million Women Study. Lancet 2003; 362:419-427.

29. Speroff L. The Million Women Study and breast cancer. Maturitas 2003;46:1-6.

30. Olson H. What can we learn from the Million Women Study. Maturitas 2003;46:1-6.

31. Velthuis-te Wierik EJM, Hendricks PT, Boerstoel-Streefland M. Clinical background of women prescribed tibolone or combined estrogen + progestogen therapies (CEPT): a UK Mediplus Study. Climacteric 2004. In press.

32. Allen DS, de Vries C, Farmer DT. Pharmaceutical content and regimen of hormone replacement therapy and risk of breast cancer. Pharmacoepidemiol Drug Saf 2002;1(Suppl 1):296-298.

33. Love CD, Muir BB, Scrimgeour JB, Leonard RC, Dillon P, Dixon J. Investigation of endometrial abnormalities in asymptomatic women treated with tamoxifen and can evaluation of the role of endometrial screening. J Clin Oncol 1999;17:2050-2054. 